livraisons

d'Histoire

de l'Architecture

\section{Livraisons de l'histoire de l'architecture}

$38 \mid 2019$

Varia III

\title{
L'Hôtel des Téléphones de Jean Boussard
}

Jean Boussard Telephone Exchange Building

Das ,Hôtel des Téléphones ' von Jean Boussard

\section{Arnaud Schoonheere}

\section{OpenEdition}

\section{Journals}

Édition électronique

URL : http://journals.openedition.org//ha/1498

DOI : $10.4000 /$ /ha. 1498

ISSN : 1960-5994

\section{Éditeur}

Association Livraisons d'histoire de l'architecture - LHA

Édition imprimée

Date de publication : 15 décembre 2019

Pagination : 75-85

ISSN : $1627-4970$

\section{Référence électronique}

Arnaud Schoonheere, "L'Hôtel des Téléphones de Jean Boussard », Livraisons de l'histoire de l'architecture [En ligne], 38 | 2019, mis en ligne le 02 janvier 2021, consulté le 06 février 2021. URL http://journals.openedition.org//ha/1498 ; DOl : https://doi.org/10.4000/lha.1498 


\section{Par Arnaud SCHOONHEERE}

\section{L'HÔTEL DES TÉLÉPHONES DE JEAN BOUSSARD}

Bon nombre de nos lecteurs connaissent la Poste de la rue du Louvre et la polémique qu'elle a engendrée au cours de ces dernières années ${ }^{1}$. En revanche, peu connaissent l'Hôtel des Téléphones qui la jouxte. Pourtant, cette construction à l'allure de forteresse émaillée constitue un jalon essentiel dans l'histoire de l'architecture publique (ill. 1). En effet, il s'agit là du premier bâtiment construit par l'État pour répondre à un programme nouveau : celui des centraux téléphoniques.

Paradoxalement aussi ostensible que méconnu, l'Hôtel des Téléphones - souvent désigné sous l'appellation de central Gutenberg - mérite que l'on s'intéresse à lui d'un peu plus près. Dans cet article, nous examinerons sa genèse en rappelant le développement notable du réseau téléphonique français à la fin du XIXe siècle, avant de nous arrêter quelques instants sur la formation et la carrière de son auteur. Puis, nous étudierons son architecture et son évolution avant de réfléchir à sa patrimonialisation.

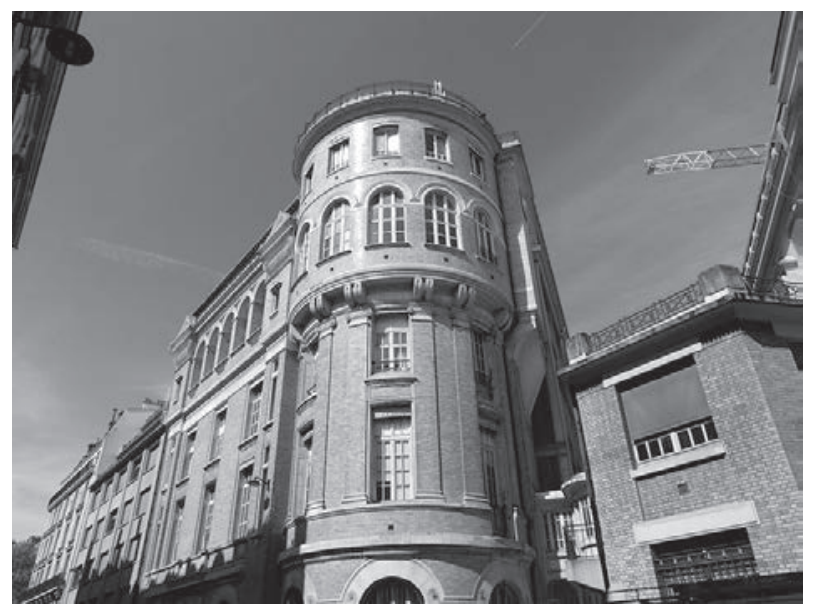

Ill. 1 : Vue actuelle de l'Hôtel des Téléphones photographié depuis la rue Jean-Jacques Rousseau (clichés de l'auteur, mai 2018). (C) D.R.

1. À ce sujet, voir : Jean-François Cabestan, «De Guadet à Perrault, la Reconversion de la poste du Louvre ", Le Moniteur, 12 septembre 2013 (en ligne). 


\section{Aux origines du réseau téléphonique parisien}

Afin de mieux comprendre les raisons qui ont amené la construction de l'Hôtel des Téléphones, il convient d'effectuer quelques rappels historiques. S'il est communément admis que l'invention du téléphone remonte à 1876 et que l'on attribue sa paternité à l'écossais Graham Bell (1847-1922), il faut attendre 1879 pour voir son introduction en France. À l'époque, au sein du ministère des postes et télégraphes, personne ne paraît soupçonner l'importance future qui sera accordée à ce nouveau moyen de communication. L'État choisit alors de remettre l'exploitation du téléphone entre les mains de compagnies privées. Cependant, il est nécessaire de préciser que cette concession - éphémère - prévoit un partage des tâches bien défini entre l'administration et les compagnies privées. À ces dernières reviennent l'installation des centraux et le raccordement des abonnés au réseau téléphonique. À l'administration revient la pose des câbles nécessaires au développement du réseau.

À Paris, comme dans d'autres villes en France, c'est la Société générale des téléphones (S.G.T.) qui détient le monopole ${ }^{2}$. Au milieu des années 1880, la S.G.T. - méfiante vis-à-vis de l'État qui souhaite reprendre la main sur l'exploitation du téléphone - cesse d'investir dans l'entretien et l'extension du réseau téléphonique. À la même époque, la notion de service public qui émerge timidement au sein du gouvernement entraîne le non-renouvellement des concessions accordées aux sociétés privées ${ }^{3}$. Finalement, l'ensemble des infrastructures nécessaires au fonctionnement des réseaux téléphoniques est nationalisé en 1889. À Paris, la situation est critique : le nombre d'abonnés croît de manière exponentielle tandis que les centraux téléphoniques saturent. Nombreux sont les Parisiens qui se retrouvent sur liste d'attente, espérant être un jour raccordés au réseau téléphonique de la capitale. L'administration des Postes et télégraphes, à laquelle est adjointe le Téléphone pour former ce qu'il convient d'appeler les P.T.T. (Postes, Téléphones et Télégraphes), décide alors la mise en place d'une commission consultative dont le but est de réfléchir à la transformation du réseau téléphonique de Paris. Dans son rapport daté du 20 novembre $1889^{4}$, la commission préconise notamment la création de quatre bureaux centraux en remplacement des douze bureaux existants, hérités de la S.G.T. L'acceptation de cette proposition conduit l'administration à construire de nouveaux bâtiments, vastes et bien éclairés, à l'instar des centraux téléphoniques que le rapporteur a pu observer à Berlin. Le premier de ces édifices à être construit est l'hôtel principal des Téléphones, que le ministère choisit d'implanter rue Gutenberg, à proximité de l'Hôtel des Postes (ill. 2). L'architecte Jean Boussard est alors désigné pour diriger les travaux.

2. Catherine Bertho (dir.), Histoire des télécommunications en France, Toulouse, Erès, 1984, p. 61.

3. Bibliothèque historique des Postes et télécommunications, Pièces et documents officiels relatifs à la question des téléphones et aux concessions de la Société Générale des Téléphones : projets de loi, traités, correspondance, TB 124.

4. Bibliothèque Historique des Postes et Télécommunications, Télégraphes et téléphones : documents, 1864-1890, TB 107. 


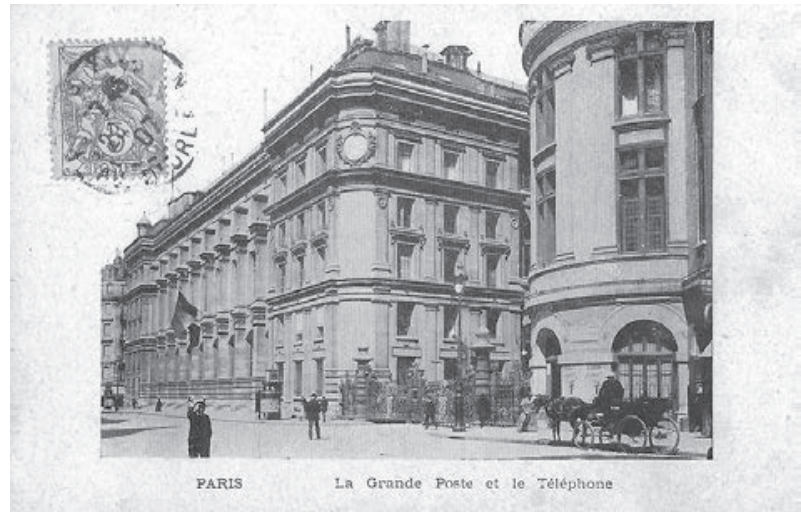

Ill. 2 : Carte postale représentant l'Hôtel des Postes et l'Hôtel des Téléphones vus depuis la rue du Louvre (collection de l'auteur). (C) D.R.

\section{Un architecte ambitieux face à un nouveau programme}

Lorsque lui est confié le projet de l'Hôtel des Téléphones, Jean Boussard (1844-1923) vient tout récemment d'être nommé architecte auprès de la direction générale des Postes et télégraphes. Originaire du département de l'Yonne, Boussard grandit au sein d'une famille aisée dont le père, médecin, exerce dans la commune de Saint-Florentin. La formation initiale de Boussard, tout comme ses années passées entre les murs de l'école des beaux-arts de Paris sont assez mal documentées. Nous savons néanmoins qu'il fréquente l'atelier d'Alexis Paccard au milieu des années $1860^{5}$ et qu'il n'a jamais concouru au traditionnel Grand Prix de Rome. Doté d'une personnalité bouillonnante, l'architecte conduit de front une carrière de constructeur et de théoricien. En effet, il ne s'illustre pas seulement dans le domaine de la construction mais également dans celui de l'édition. Il publie ainsi plusieurs ouvrages, dont le premier - et certainement l'un des plus importants au vu de sa diffusion - est un recueil de tombeaux, paru en $1870^{6}$. Ce recueil, constitué d'une cinquantaine de planches gravées, s'inscrit dans une tradition éditoriale lancée au début du XIX ${ }^{\mathrm{e}}$ siècle ${ }^{7}$.

Au-delà de ses recueils d'architecture dans lesquels il esquisse brièvement ses théories en matière de construction, Boussard publie un important ouvrage intitulé L'Art de bâtir sa maison (1887) où il consigne les règles à suivre pour l'édification de la demeure idéale, à la fois rationnelle, économique et hygiénique, qu'il imagine

5. Arch. Nat., dossier $\mathrm{AJ}^{52} 357$.

6. Jean Boussard, Recueil de tombeaux les plus remarquables exécutés de nos jours et représentés en perspective, Paris, Librairie polytechnique de J. Baudry, 1870.

7. Citons les ouvrages de César Daly, Architecture funéraire contemporaine. Spécimens de tombeaux, chapelles funéraires, mausolées, 1871 et de Louis Normand, Monuments funéraires choisis dans les cimetières de Paris et les principales villes de France, 1847. 
calquée sur le modèle de villas gallo-romaines du $\mathrm{III}^{\mathrm{e}}$ siècle. De cet ouvrage et des théories qu'il renferme naîtront plusieurs habitations réparties sur l'ensemble du territoire français ${ }^{8}$. D'emblée, l'ambitieux Boussard cherche à s'imposer parmi les plus grands architectes de son temps. En dehors des immeubles de rapport et des constructions luxueuses qu'il réalise pour une clientèle privée, Boussard parvient à accéder à la commande publique lorsqu'il intègre l'administration des Postes et Télégraphes, d'abord au grade d'inspecteur des travaux, puis à celui d'architecte en chef $^{9}$. Pour cette administration, il va d'abord construire plusieurs hôtels des postes en province ${ }^{10}$ avant de concevoir les plans des premiers centraux téléphoniques parisiens. Parmi ces derniers, l'Hôtel des Téléphones fait figure d'exception, d'abord par la place qu'il ambitionne d'occuper dans le maillage du réseau téléphonique, ensuite par sa typologie.

\section{La construction de l'Hôtel des Téléphones}

À l'instar de l'Hôtel des Postes qu'il jouxte, l'édification de l'Hôtel des Téléphones a produit une abondante littérature, qu'il s'agisse de la presse à grand tirage ou des revues spécialisées d'architecture. Cette construction est donc exceptionnellement bien documentée. Fort heureusement, les Archives de Paris conservent le permis de construire de ce central téléphonique. Le dossier qui le compose nous renseigne sur la date et les modalités de dépôt des plans de la nouvelle bâtisse ${ }^{11}$. On apprend que la demande de permis de construire est déposée par Boussard lui-même au nom de l'État le 9 octobre 1890. Dans le dossier, il est précisé que l'architecte cherche à obtenir "la permission de construire un monument public destiné à contenir le "Poste principal téléphonique", conformément aux plans produits, lequel sera élevé "d'un rez-de-chaussée et de 3 étages sous comble" ". Malheureusement, les originaux des plans, établis sur papier calque, sont en trop mauvais état pour pouvoir être consultés. Nous ignorons donc la date à laquelle Boussard a pu établir les plans définitifs de son bâtiment. Néanmoins, force est de constater que l'architecte a bénéficié d'assez peu de temps pour la réalisation de ses plans. En effet, entre la nationalisation du réseau téléphonique et le dépôt du permis de construire il ne s'écoule qu'un peu plus d'un an. Sans doute Boussard s'est-il efforcé de respecter au mieux les préconisations établies par le rapport de la commission évoquée précédemment.

8. Boussard a en effet construit de nombreuses villas, tant en région parisienne qu'en province. Deux édifices retiennent particulièrement notre attention : la Maison romaine d'Épinal (Vosges), et une villa située avenue Flachat à Asnières (Hauts-de-Seine) qui a conservé la quasi-totalité de son décor d'origine.

9. Arch. Nat., dossier $\mathrm{F}^{90} 20513$.

10. Figurent notamment dans cette liste, les hôtels des postes d'Angers (1887), Bordeaux (1892), Fontainebleau (1893) et Orléans (1898).

11. Arch. Paris, dossier VO ${ }^{11} 1615$. 

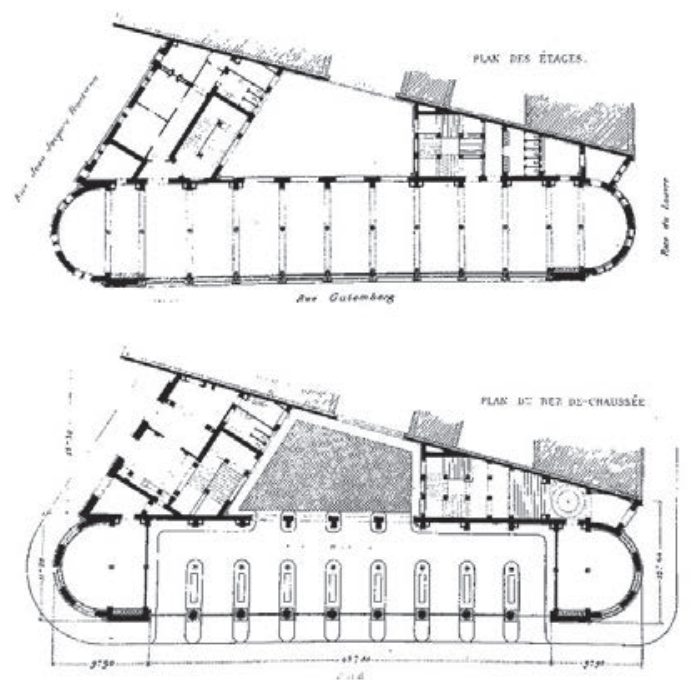

Ill. 3 : Plans des différents niveaux de l'Hôtel des Téléphones tirés de la revue La Semaine des constructeurs en date du 2 juillet 1892. (C) D.R.

Sur une parcelle étroite de près de 1200 mètres carrés, l'architecte prévoit l'installation d'un long bâtiment formé de deux ailes (ill. 3). L'articulation de celles-ci génère une petite cour intérieure dont l'usage est réservé à l'Hôtel des Postes qui y trouve l'espace idéal pour le remisage de ses voitures hippomobiles. La première aile, longue d'une soixantaine de mètres, borde la rue Gutenberg. Elle est flanquée de deux tours réalisées en maçonnerie de pierres et de briques émaillées qui conferent à l'édifice un aspect de forteresse médiévale. Cette partie du bâtiment étant destinée à accueillir, superposées, les salles des multiples où s'affaireront les "demoiselles du téléphone ${ }^{12}$ ", l'architecte cherche à y apporter un maximum de lumière. Il conçoit alors une façade toute de fer et de verre à l'instar de celles qui garniront, près d'une dizaine d'années plus tard, les sous-stations électriques de la Compagnie générale du Métropolitain. La rue Gutenberg étant trop étroite pour pouvoir apprécier avec suffisamment de recul la façade du central téléphonique, seules les vues d'artistes insérées dans les revues de l'époque nous permettent de considérer l'effet que pouvait produire cette construction sur le promeneur. On y constate que la façade de verre et de fer revêt un aspect de légèreté, souligné par la série d'arcades du premier niveau. Elle vient alors contrebalancer la lourdeur et la massivité de la seconde aile, érigée le long de la rue Jean-Jacques Rousseau. De dimensions beaucoup plus modestes, celle-ci accueille les bureaux et une partie des espaces de circulation du central téléphonique à l'instar du grand escalier de service.

12. Avant son automatisation, le central téléphonique Gutenberg accueillait quotidiennement plusieurs centaines de téléphonistes. À ce sujet, lire : Madeleine Campana, La Demoiselle du Téléphone, Paris, Jean-Pierre Delarge, 1976. 


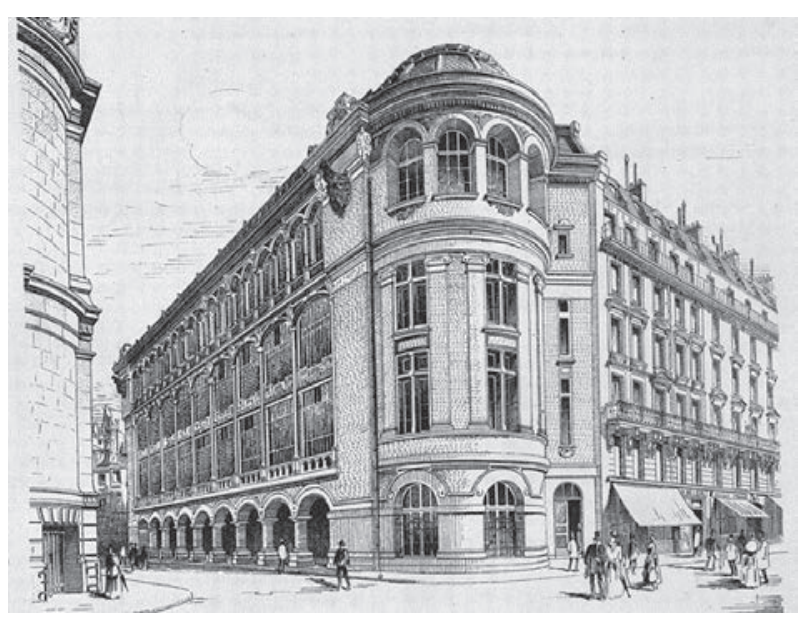

Ill. 4 : Vues en perspective de l'Hôtel des Téléphones tirées de la revue La Construction moderne en date du $1^{\text {er }}$ avril 1893 (planche 53). (C) D.R.

Ce dernier, construit en béton, est largement ouvert sur l'extérieur par une série de fenêtres à arcs rampants dont la silhouette ne va pas sans évoquer celle des cages d'escaliers des châteaux de la Renaissance ${ }^{13}$.

L'autorisation de bâtir obtenue en janvier 1891, la construction débute en avril de la même année et se poursuit jusqu'à l'automne 1892, lorsque le monument est livré à l'État. La presse se hâte alors de couvrir l'événement ${ }^{14}$.

Dans la revue La Construction moderne en date du $1^{\text {er }}$ avril $1893^{15}$ paraissent d'intéressantes vues en perspective de l'Hôtel des Téléphones (ill. 4). En quelques lignes, la genèse ainsi que la description du bâtiment sont données. Il y est rappelé que " par suite de l'extension donnée au service téléphonique à Paris, la construction d'un Hôtel central des Téléphones a été reconnue nécessaire ". L'auteur ajoute que « c'est M. Boussard, architecte, qui a été chargé d'élever ce monument d'un genre tout nouveau ». Cette dénomination de monument d'un genre nouveau confirme l'aspect inédit que revêt la construction d'un central téléphonique. L'auteur, anonyme, souligne également que "l'originalité de la construction consiste principalement dans l'emploi de la céramique pour les façades». Ces céramiques, qui incluent les briques émaillées employées pour l'appareil des murs donnant sur les rues du Louvre et Jean-Jacques Rousseau ainsi que les encadrements de terre cuite installés autour des différentes baies, proviennent d'une entreprise chère à Boussard :

13. Nous pensons notamment au château de Châteaudun auquel Boussard a consacré une étude historique dans la revue Le Moniteur des architectes. Cette remarque pertinente nous a été communiquée par l'un de nos relecteurs, M. Thibault Le Hégarat.

14. L'article tiré du journal Le Monde illustré en date du 5 novembre 1892 est de notre point de vue le plus complet : Guy Tomel, "L’Hôtel des Téléphones ", Le Monde illustré, n 1858, 5 novembre 1892, p. 291-294.

15. Anonyme, «L’Hôtel des Téléphones, à Paris ", La Construction moderne, nº 26, $1^{\text {er }}$ avril 1893, p. 309. 


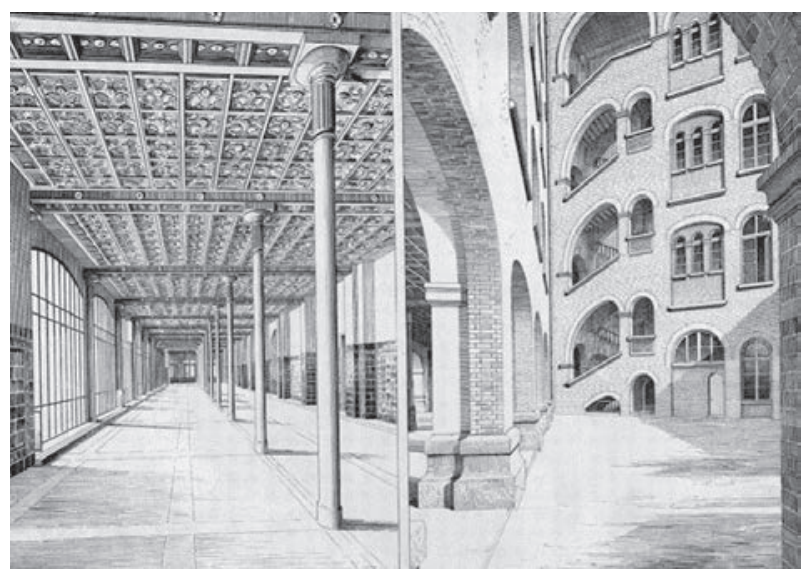

Ill. 5 : Vues en perspective de l'Hôtel des Téléphones tirées de la revue La Construction moderne en date du $1^{\text {er }}$ avril 1893 (planche 54). (C) D.R.

les usines Perrusson d’Écuisses, en Saône-et-Loire ${ }^{16}$. Résistantes aux effets néfastes de la pollution (elles ne se couvrent pas de croûte noire comme la pierre calcaire en raison de leur émail) ces céramiques ont assuré à l'Hôtel des Téléphones des façades colorées et rutilantes tout le long du XXe siècle. Étonnamment, aucun journal à l'époque ne semble avoir condamné ce parti pris esthétique, en rupture totale avec les autres immeubles du quartier.

Si les choix de Boussard peuvent surprendre, il faut néanmoins souligner la cohérence du programme et de la construction. D’abord, le recours aux matériaux industriels tels que le fer et le verre convient parfaitement à la nature du lieu et répond aux besoins de luminosité que nécessite cette "usine" téléphonique. En outre, sa volonté de rupture et de modernité s’inscrit également dans la maçonnerie : par le recours à une brique émaillée de couleur claire qui tranche avec la pierre des immeubles contigus; mais également par la présence des deux rostres qui flanquent la façade vitrée, à hauteur du troisième étage. Celles-ci pourraient vouloir signifier aux passants de la rue du Louvre que le bâtiment qui se dresse devant eux, constitue le vaisseau amiral d'une flotte conquérante, allégorie de l'administration des P.T.T. Ajoutons que le rationalisme de l'édifice est maintes fois souligné dans les revues spécialisées de l'époque, à l'instar de La Semaine du constructeur qui déclare à ce sujet: "le principe d'accusation au-dehors de la distribution intérieure se trouve rigoureusement appliqué ${ }^{17}$ ". Ce rationalisme, visible en élévation, s'exprime également dans les volumes intérieurs où de vastes espaces sont conçus pour l'installation du matériel téléphonique (ill. 5).

16. À ce sujet, lire : Luc Dunias, Les Perrusson-Desfontaines : industriels céramistes et leur résidence d'Écuisses, Le Creusot, Éd. Écomusée Creusot Montceau, 2004, p. 63-64.

17. F. Monmory, "Poste central téléphonique ", La Semaine des constructeurs, n 13, 17 septembre 1892, p. 151. 
Enfin, le plan en équerre de l'Hôtel des Téléphones, que nous évoquions plus haut, jouit d'une descendance exceptionnelle. Ainsi, l'architecte François Le Cœur (1872-1934), qui travailla également au service des P.T.T., employa à son tour cette formule pour plusieurs de ses édifices dont le central téléphonique Bergère situé rue du faubourg Poissonnière ${ }^{18}$. De même, Paul Guadet (1873-1931) conserva cette formule lors de l'édification de ses centraux téléphoniques parisiens.

\section{Un édifice rescapé et remanié}

Malgré ses qualités, l'Hôtel des Téléphones de la rue du Louvre a pourtant bien failli disparaître. Le 20 septembre 1908 au soir, un incendie ravage le bâtiment (ill. 6). Là encore, la presse s'empare du sujet et les articles qui en découlent se comptent par dizaines. Fait remarquable, Boussard livre son point de vue sur l'évènement dans une interview qu'il accorde au journal Le Rappel ${ }^{19}$. L'architecte fait part de son incompréhension et précise : "Les salles n'avaient d'autre communication entre elles que la cheminée qui servait au passage des câbles. Il était donc facile d'isoler une salle dans laquelle un incendie se déclarait. Pour moi, c'est une chose inconcevable que la destruction totale de l'Hôtel des Téléphones. " Boussard ajoute qu'à l'époque où il a conçu son bâtiment, "l'administration ne voulait pas entendre parler du ciment armé. ${ }^{20}$ [...] On était donc obligé d'employer, pour soutenir le plancher d'aussi vastes salles, des colonnes de fer. Ces colonnes auront sans doute, sous l'action d'un incendie qui a duré plusieurs heures, rougi et se seront déformées sous le poids. Dans ce cas tout l'édifice est compromis. " Et l'architecte est loin de se méprendre. En effet, les photographies prises au lendemain de l'incendie attestent du ploiement des planchers et de l'éclatement de plusieurs colonnes (ill. 7). De plus, si les propos que l'on prête à Boussard se révèlent exacts, il est nécessaire de souligner le fait que l'architecte se montre novateur sur le plan technique. Ainsi exprime-t-il sa volonté, très précoce, d'employer le ciment armé en lieu et place du fer et de la fonte dans la construction des planchers et la structure du bâtiment. Malheureusement, force est de constater que Boussard n'utilisera le ciment armé qu'à de très rares occasions dans ses constructions ultérieures ${ }^{21}$.

Malgré l'importance des dégâts matériels, la décision est prise par l'administration de ne pas reconstruire l'intégralité du bâtiment. Les raisons qui ont amené

18. L'édifice, dont la construction débute en 1912, se situe à l'angle de deux voies : la rue Bergère, le long de laquelle est érigée l'aile abritant les salles de multiples, et la rue du faubourg Poissonnière, sur laquelle donne l'aile en retour d'équerre abritant les bureaux.

19. Anonyme, "L'Incendie de l'Hôtel des Téléphones ", Le Rappel, 24 septembre 1908, p. 2.

20. C'est en effet à François Le Cœur que les historiens attribuent l'introduction du ciment et du béton armé dans les bâtiments publics sous contrôle de l'administration des P.T.T. À ce sujet, Isabelle Carreau-Vacher, "François Le Cour, un pionnier du ciment armé en France ", Colonnes, no 10, novembre 1997 , p. 12-20.

21. Était-ce donc un mensonge ou de la provocation? L'état actuel de nos recherches ne permet pas de trancher la question. 


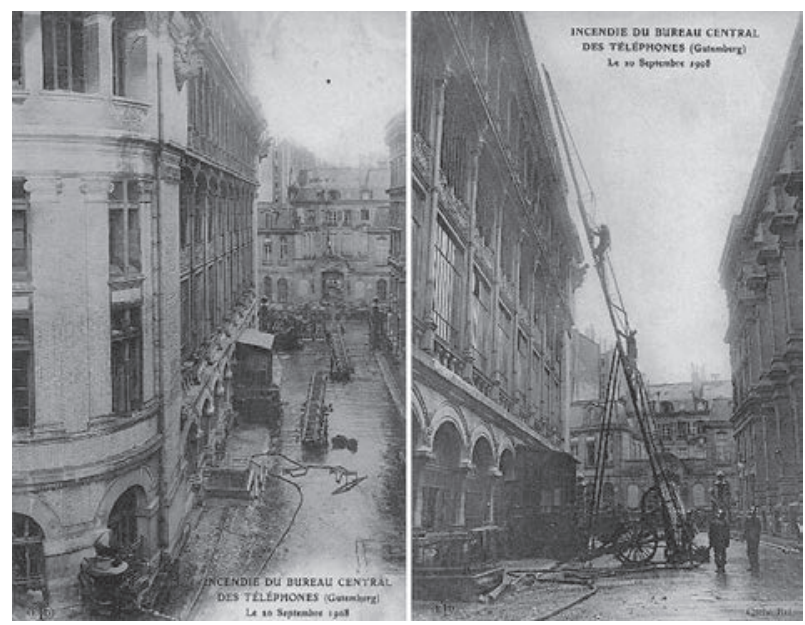

Ill. 6 : Cartes postales représentant l'incendie de l'Hôtel des Téléphones à l'automne 1908 (collection de l'auteur). (C) D.R.

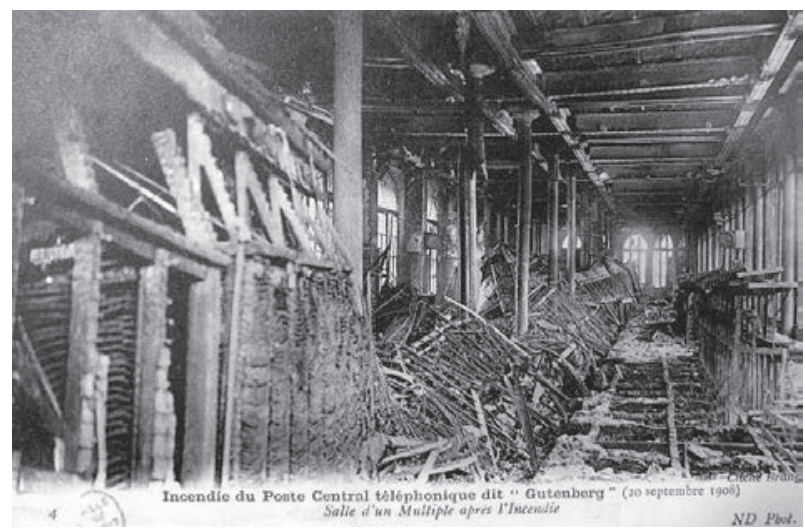

Ill. 7 : Carte postale représentant une salle de l'Hôtel des Téléphones après l'incendie survenu à l'automne 1908 (collection de l'auteur). (c) D.R.

cette décision sont de différents ordres : à la fois pratiques et financiers. Par chance, l'incendie a épargné une partie des installations techniques situées au premier étage du bâtiment. Aussi, le central téléphonique provisoire installé dans la cour qui sépare l'ancien Hôtel des Téléphones de l'Hôtel des Postes peut-il être raccordé rapidement au matériel épargné afin de léser le moins possible les abonnés. La conservation d'une partie du bâtiment incendié s'est alors imposée à l'Administration. De surcroît, la reconstruction intégrale d'un pareil édifice aurait fortement obéré les finances de l'État. La solution d'une reconstruction partielle - donc, à moindre coût - est d'autant plus souhaitable. C'est l'architecte Charles Giroud (1871-1955) qui est chargé de la reconstruction du central téléphonique. La demande de permis 
de construire est déposée le 22 novembre 1909 par M. Le Directeur des services téléphoniques $^{22}$. Le dossier vise à obtenir la permission de reconstruire l'Hôtel des Téléphones « en conservant une partie du bâtiment sinistré ». Les plans, datés du 11 novembre 1909, attestent que l'aspect général du bâtiment original est conservé à ceci près que le nouvel édifice est surélevé d'un étage installé en encorbellement et que les planchers métalliques laissent place à des planchers en béton armé. Finalement, le nouvel édifice, inauguré en 1912, conserve le souvenir prégnant de Jean Boussard. Outre quelques aménagements intérieurs nécessaires compte tenu de l'évolution des technologies et de la miniaturisation du matériel électrique, le central téléphonique Gutenberg tel que l'on peut l'observer aujourd'hui, a dans l'ensemble conservé ses dispositions d'origine, tant en plan qu'en élévation.

\section{Quel avenir pour ce "monument"?}

L'Hôtel des Téléphones érigé par Jean Boussard constitue, nous l'avons vu, un édifice important dans l'histoire de l'architecture publique. Pourtant, l'édifice demeure absent des manuels d'histoire de l'art spécialisés, à de rares exceptions près ${ }^{23}$. Seul le chercheur américain Frances H. Steiner a vu dans l'Hôtel des Téléphones "l'un des plus brillants exemples de fonctionnalisme au XIX siècle $^{24}{ }^{2}$. Par l'intermédiaire de cette étude, nous souhaitions donc rendre à ce bâtiment et à son auteur les honneurs qu'ils méritent.

Lorsque Jean Boussard meurt le 14 juin 1923 dans son appartement de la rue Ribera (Paris, $\mathrm{XVI}^{\mathrm{e}}$ arrondissement), il ne laisse aucun héritier. Ses archives, dispersées ou bien détruites, ne sont pas parvenues jusqu'à nous. Aussi, rares sont les historiens de l'art qui ont pris le temps de s'intéresser à cet architecte et à son œuvre. Aucune monographie, ni même aucun article scientifique ne lui ont jamais été dédiés. Aujourd'hui, cette lacune est en voie d'être comblée : d'abord, par la publication de cet article qui, nous l'espérons, donnera lieu à d'autres études monographiques; ensuite, par la rédaction de notre thèse portant sur la carrière de Boussard. Les travaux que nous menons actuellement sont complexes, compte tenu de l'absence de fonds d'archives légué par l'architecte. Il nous est nécessaire de voyager dans tout l'hexagone et d'exercer notre oil pour faire connaitre la diversité et l'abondance de la production de Jean Boussard.

Face à la pression immobilière, l'Hôtel des Téléphones - propriété de l'entreprise Orange - paraît voué à la vente et à la reconversion à l'instar d'un grand

22. Arch. Paris, dossier VO ${ }^{11} 1937$.

23. Le bâtiment a fait l'objet de rares notices essaimées dans différents ouvrages tels que : Marie-Françoise Laborde, Architectures industrielles, Paris, Parigramme, 2003, p. 18.

24. Frances H. Steiner, French Iron Architecture, Ann Arbor, Mich, UMI research press, 1984, p. 113, " [...] one of the clearest examples of functional form of the nineteeth century ". 
nombre d'anciens centraux téléphoniques ${ }^{25}$. Ce contexte doit nous inciter à faire preuve d'une extrême vigilance, laquelle est d'autant plus justifiée que l'immeuble n'est protégé que par le plan local d'urbanisme (P.L.U.) de la Ville de Paris. Or, l'édifice nous parvient dans un état historique intéressant, offrant encore à l'œil du visiteur quelques reliquats du premier central téléphonique tels que la cage d'escalier, les colonnes en fonte du premier niveau, les façades de briques émaillées, etc., ainsi que des aménagements postérieurs hérités des travaux de Charles Giroud. Ne bénéficiant d'aucune protection au titre des Monuments historiques, l'Hôtel des Téléphones pourrait être aux prises avec toutes sortes de déprédations visant à retirer les derniers éléments décoratifs qu'il renferme et qui témoignent de son glorieux passé. Aussi interrogeons-nous : la protection au titre du PLU ${ }^{26}$ est-elle suffisante et pérenne? Rien n'est moins sûr...

In fine cet article revêt la forme d'un plaidoyer en faveur de la sauvegarde de ce monument insigne de l'histoire des télécommunications françaises. On aimerait faire reconnaitre les valeurs artistique et historique de ce lieu qui fut jadis le fleuron d'une jeune administration et d'un service public en plein essor sous la Troisième République.

Arnaud SCHOONHEERE

Doctorant à l'École pratique des hautes études

25. Citons pour exemple le central téléphonique "Ségur ", situé avenue de Saxe et reconverti en hôtel de luxe.

26. Plan Local d'Urbanisme de la Ville de Paris adopté les 12 et 13 juin 2006, disponible en ligne sur https://capgeo.sig.paris.fr/Apps/ParisPLU/ et consulté le 25 juin 2018. 
